\title{
Caracterización cariotípica en mitosis y meiosis del robalo blanco Centropomus undecimalis (Pisces: Centropomidae)
}

\author{
Lenin Arias-Rodriguez ${ }^{1 *}$, Jeane R. Indy ${ }^{1,2}$, Rosa I. Ahumada-Hernández ${ }^{1}$, Helen Barragán- \\ Cupido $^{1}$, Antonieta A. Ávalos-Lázaro ${ }^{1} \&$ Salomón Páramo-Delgadillo ${ }^{1}$ \\ 1. División Académica de Ciencias Biológicas, Universidad Juárez Autónoma de Tabasco (UJAT), C.P. 86150, \\ Villahermosa, Tabasco, México; leninariasrodriguez@hotmail.com, rosaiselaahumada@hotmail.com, \\ helenbarragan@hotmail.com, abisag_avalos@hotmail.com; salomonparamo@hotmail.com \\ 2. Faculty of Fisheries and Marine Sciences, Sam Ratulangi University, Kampus Bahu, (UNSRAT). P.O. 95115. Manado, \\ Indonesia; jeanerimberindy@yahoo.com \\ * Correspondencia.
}

Recibido 19-V-2010. Corregido 01-XII-2010. Aceptado 10-I-2011.

\begin{abstract}
Karyotypic characterization in mitosis and meiosis of the common snook Centropomus undecimalis (Pisces: Centropomidae). The common snook Centropomus undecimalis inhabits marine, brackish and freshwater habitats in the Western Central Atlantic Ocean, including the Gulf of Mexico. Common snook is an economically important fish in many localities, nevertheless the number of studies on its biology and genetics are still few. The present study attempts to establish the cytogenetic profiles of the specimens collected in Paraiso Municipality Tabasco, Mexico. Tissue of five females and eight male organisms were processed by conventional cytological techniques to obtain chromosome slides of high quality in order to assemble the karyotype. The results from the kidney tissue analysis showed that $85.1 \%$ of 288 mitosis had a $2 n=48$ chromosomes, and $52.8 \%$ of 104 meiosis exhibited the haploid number $1 \mathrm{n}=24$. The diploid karyotype showed 48 monoarmed chromosomes of the telocentric (T) type. There was no chromosome heteromorphism between females and males. The diploid karyotype was very similar to that observed in the majority of marine fishes. Rev. Biol. Trop. 59 (2): 683-692. Epub 2011 June 01.
\end{abstract}

Key words: karyotype, chromosome, common snook, Centropomus undecimalis.

Los robalos, como se les conoce coloquialmente a los miembros de la familia Centropomidae, han sido recientemente clasificados en un sólo género: Centropomus (Nelson 2006). De acuerdo con Rivas (1986) y Nelson (2006), los Centropomus han sido descritos como un grupo compacto de peces que es muy homogéneo, gran parte de su vida se da en los mares del continente Americano y un periodo reducido en cuerpos de agua dulce, por ello tienen un alto grado de diferenciación, si se les compara con los miembros de la familia hermana Lates y Psammoperca de la región Indo-Pacifico y África. De revisiones taxonómicas previas
(Rivas 1986) y bioquímicas actuales (Tringali et al. 1999), se ha logrado demostrar que ni una sola de las especies de Centropomus que habitan en el océano Atlántico viven en el océano Pacifico o viceversa.

Los robalos en el continente Americano, están representados por 12 especies nominales (Rivas 1986, Nelson 2006). Seis especies de robalo (C. medius, $C$. nigrescens, $C$. viridis, $C$. unionenses, $C$. armatus y $C$. robalito) son reconocidas en el Pacifico oriental y su rango de distribución está contemplado desde el Golfo de Baja California en el norte de México hasta las costas de Perú en Sudamérica (Rivas 1986, 
Nelson 2006). Por otro lado, seis especies han sido reconocidas para el Atlántico occidental, C. pectinatus, C. undecimalis, $C$. poeyi, $C$. parallelus, $C$. mexicanus y $C$. ensiferus (Rivas 1986), con un rango de distribución general como grupo, entre la península de Florida en los Estados Unidos de Norte América y en Sudamérica hasta las costas de Brasil, incluyendo las islas Antillas (Marshall 1958, Volpe 1959, Chávez 1963, Rivas 1986, ÁlvarezLanjonchére \& Suzuki 2008). La mayoría de las especies de robalo del continente Americano, tienen distribución amplia a lo largo de las aguas del océano Pacifico y Atlántico, siendo $C$. poeyi, la única especie con rango reducido de distribución tan sólo a lo largo de las costas del Golfo de México en la sonda de Campeche del Océano Atlántico (Chávez 1963).

En general, todas las especies de robalo son consideradas como peces de alto valor económico, principalmente por el precio que alcanzan en los mercados debido al sabor de la carne y al tamaño que alcanzan en etapa adulta (Tucker Jr. 1987, Tringali et al. 1999, Perera-García et al. 2008). El robalo blanco ó C. undecimalis, es una de las especies de mayor importancia económica debido a los volúmenes de captura que se registran a lo largo del año (Tucker Jr. 1987, Tringali et al. 1999, PereraGarcía et al. 2008).

Aunque los centropómidos, son uno de los peces con mayor importancia comercial, la información sobre su biología y genética es muy limitada (Marshall 1958, Volpe 1959, Chávez 1963, Rivas 1986, Miller et al. 2005, Álvarez-Lanjonchére \& Suzuki 2008, PereraGarcía et al. 2008). La mayoría de los estudios en el grupo, se han realizado básicamente sobre aspectos de taxonomía, distribución (Rivas 1986, Tucker Jr. 1987, Chávez 1963) y recientemente sobre el estado actual de sus pesquerías (Perera-García et al. 2008). En los estudios de taxonomía y distribución, se ha detectado un alto grado de traslape tanto en los caracteres merísticos y morfométricos empleados para la separación de las especies, como también de los hábitats, sitios de reproducción y áreas de migración (Rivas 1986, Miller et al. 2005), lo que sugiere la posibilidad de hibridación o presencia de genotipos especiales que pueden ser atribuidos a razas o variedades de interés biológico y acuícola que pueden ser asociados a sólo un número reducido de auténticas especies.

Los estudios recientes de genética en el grupo (Tringali et al. 1999, Peregrino-Uriarte et al. 2007), muestran de igual modo un probable traslape entre los loci proteicos y moleculares (p.e. ADN $m$ y microsatelital) que probablemente son indicadores que sugieren presencia de especies híbridas. Sin embargo, las evidencias no han sido lo suficientemente circunstanciales para probar la citada hipótesis, debido a la carencia de estudios citogenéticos y experimentos de hibridación artificial, la cual esta limitada por la carencia de metodologías que permitan la reproducción controlada en el grupo.

Las evidencias de tipo citogenético, son pocas en los robalos y solo existen bajo el contexto de literatura gris, con poco acceso a los datos y análisis reportados, entre ellos se encuentra el trabajo de citogenética de Barreto-Neto (2001), para C. mexicanus y $C$. undecimalis. En suma debido a los argumentos anteriores, el objetivo principal de este trabajo fue el de caracterizar por el método convencional de citogenética los cromosomas en mitosis y meiosis de la población de robalo blanco $C$. undecimalis que habita en la región costera del Golfo de México en Tabasco, México.

\section{MATERIALES Y MÉTODOS}

\section{Recolecta de especímenes e identificación} taxonómica: Cinco hembras y ocho machos de robalo blanco $(25.1 \pm 8.9 \mathrm{~cm}, 90.9 \pm 9.2 \mathrm{~g})$ en edad adulta, fueron recolectados durante los meses de octubre-noviembre del 2008 en la laguna costera Mecoacán, que se encuentra ubicada en el municipio de Paraíso, Tabasco, en el sureste de México. La identificación taxonómica, siguió los caracteres merísticos y morfométricos clásicos que recomienda Rivas (1986) y Miller et al. (2005).

Procedimiento citológico y elaboración de preparaciones cromosómicas: Los 
especímenes fueron tratados con ligeras modificaciones conforme al procedimiento empleado por Arias-Rodriguez et al. (2007, 2008, 2009), como se describe a continuación: Los especímenes, fueron tratados con una solución acuosa de colchicina $(28 \mu \mathrm{g} / \mathrm{g}$ de peso) disuelta en citrato de sodio al $0.1 \%$, inyectada en la aleta dorsal y en la región intraperitoneal $(50 \% / 50 \%)$. Treinta minutos después, los tejidos de interés: branquias, riñón y gónadas fueron removidos y transferidos a colchicina disuelta al $0.1 \%$ en citrato de sodio al $0.1 \%$. Después, fueron incubados a $37.0 \pm 1.0^{\circ} \mathrm{C}$ durante cinco horas. Posteriormente, disgregados, hidratados $\left(37.0 \pm 1.0^{\circ} \mathrm{C}\right)$ por $60 \mathrm{~min}$ en citrato de sodio al $2.0 \%$ y prefijados (durante $96 \mathrm{hrs} / 4^{\circ} \mathrm{C}$ ) por adición (en proporción similar a la del agente hidratante 1:1) del fijador preparado a partir de una mezcla de metanol $\left(4^{\circ} \mathrm{C}\right)$ con ácido acético (4:1).

Los tejidos, fueron fijados reemplazando el prefijador por el fijador 4:1, mediante el centrifugado a 6000r.p.m. durante 15 minutos a $4^{\circ} \mathrm{C}$ (Eppendorf Centrifugue 5810-R); dicha operación fue repetida hasta que los tejidos tomaron coloración blanquecina, inmediatamente fueron colocados a $4^{\circ} \mathrm{C}$ y desde ese momento solo se emplearon durante un mes.

La solución celular $\left(\mathrm{a} 4^{\circ} \mathrm{C}\right)$ derivada de cada tejido, fue goteada sobre series de portaobjetos que fueron colocados en etanol absoluto a $4^{\circ} \mathrm{C}$ y desde $1.70 \mathrm{~m}$. Las preparaciones cromosómicas, fueron secadas con la flama de un mechero de alcohol y teñidas conforme a las recomendaciones de Denton (1973) y Kligerman \& Bloom (1977) con giemsa al $10 \%$ por $30 \mathrm{~min}$.

El sexo de los especímenes, se determinó por la técnica de aplastado de una porción de gónada, tinción con solución madre de giemsa y observación bajo el microscopio con los objetivos 10X y 40X (Arias-Rodriguez et al. 2008, 2009).

Análisis microscópico y elaboración del cariotipo: El análisis y selección de las mejores dispersiones cromosómicas, se realizó con los objetivos 40X y 100X del microscopio Zeizz
Axiostar-Plus. Las mejores dispersiones cromosómicas (con cromosomas menos encimados y bien dispersos) fueron foto-digitalizadas con una cámara Sony Cybershot (DSC-W30).

El número cromosómico modal diploide, se estimó con base en el análisis de frecuencia de cromosomas mostrado en las imágenes digitalizadas, fotos impresas en alta resolución y por conteo directo con el microscopio óptico. Nueve fotografías en estadio mitótico y tres en estadio meiótico, se ampliaron e imprimieron en alta resolución para emplearse como base para elaborar el cariotipo.

El arreglo y clasificación del cariotipo en meiosis y mitosis, tuvo como referencia la propuesta metodológica de Levan et al. (1964), para ello se tomaron las medidas de longitud total del brazo q (brazo largo) de cada cromosoma en micrómetros $(\mu \mathrm{m})$, se calculó también el valor medio, la desviación estándar y la longitud relativa de cada par de cromosomas [longitud de cada par de cromosomas q/ longitud total del complemento cromosómico diploide (100)]. Los cromosomas individuales, del cariotipo representativo en ambos estadios celulares (mitosis y meiosis), fueron recortados electrónicamente con el programa Photoshop CS 8.0.1 (Adobe ${ }^{\circledR}$ ) y ordenados manualmente con base en su longitud.

Los datos, de las medidas promedio de cada par cromosómico (en $\mu \mathrm{m}$ ) de los cariotipos en mitosis y meiosis, fueron evaluadas por las pruebas de normalidad y homocedasticidad $(\mathrm{p}<0.05)$ (Zar 1984). Posteriormente, los pares cromosómicos entre los cariotipos mitóticos y meióticos fueron comparados por un análisis de varianza (ANVA) de dos vías a $\mathrm{p}<0.05$ de significancia (Zar 1984). Las diferencias en longitud $(\mu \mathrm{m})$ de cada par de cromosomas (brazo largo q) entre estadios (mitosis/meiosis) se estimó por la comparación múltiple de Tukey $(\mathrm{p}<0.05)$ (Zar 1984). El número fundamental (NF), se estableció conforme al número de brazos cromosómicos del complemento cromosómico diploide y el arreglo del ideograma en mitosis y meiosis se fundamentó en la longitud de brazos q en $\mu \mathrm{m}$ (Denton 1973). 


\section{RESULTADOS}

Ciento veinte preparaciones cromosómicas, con dispersiones metafásicas de buena calidad, fueron elaboradas de muestras de tejido gonadal (30) y riñón (90) de trece especímenes de robalo blanco $C$. undecimalis (cinco hembras y ocho machos). Las preparaciones elaboradas con tejido de las branquias, no mostraron metafases mitóticas suficientes y de calidad por lo que fueron descartadas para el estudio.

Se contabilizaron un total de 392 metafases, siendo 288 de los campos cromosómicos mitóticos (riñón) y 104 meióticos (gónadas). Del análisis de frecuencias de las metafases observadas, los datos mostraron que el $85.1 \%$ (245 metafases) de las dispersiones mitóticas corresponde a una moda de $2 \mathrm{n}=48$ cromosomas (Fig. 1A). Mientras el análisis de 104 campos
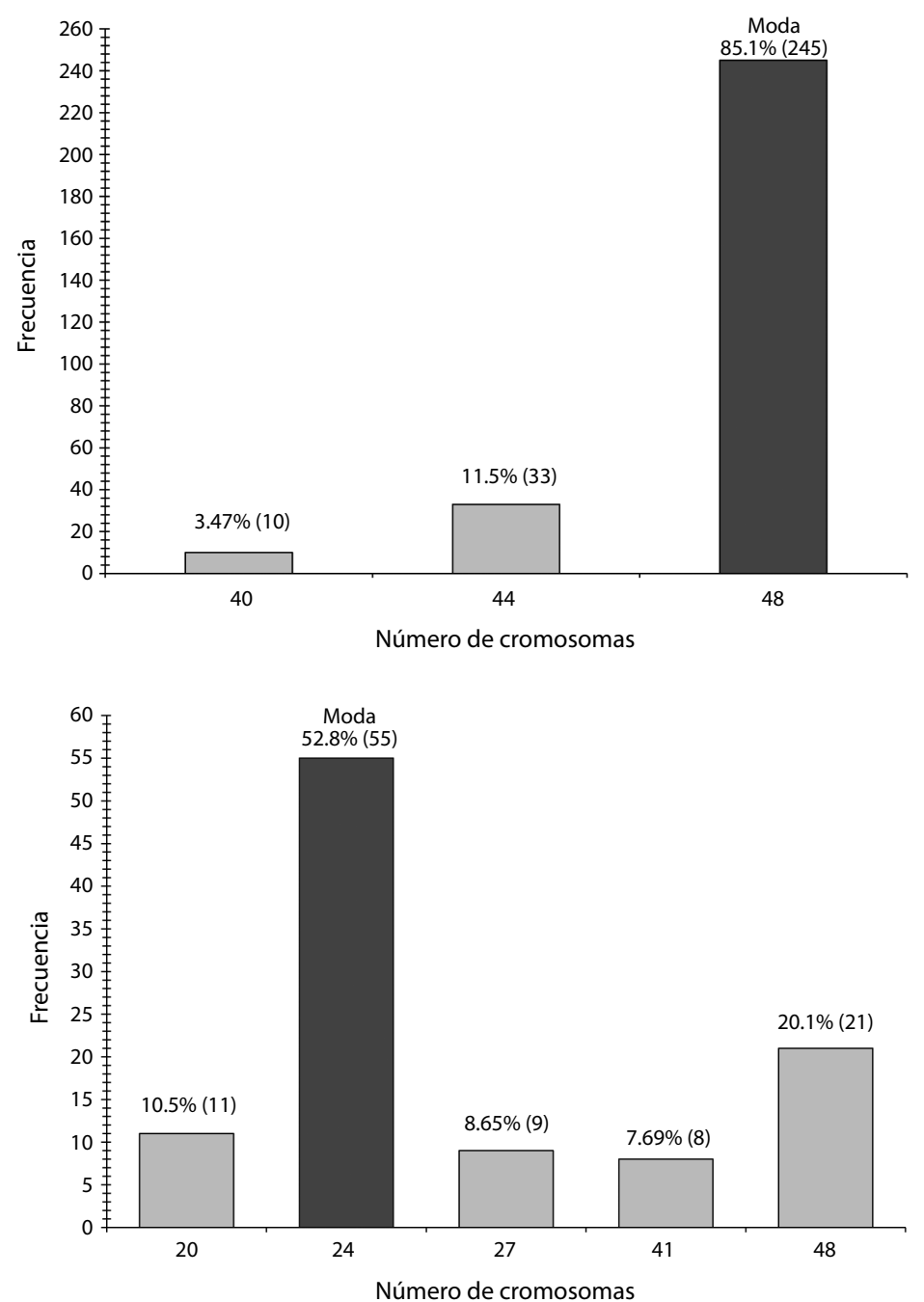

Fig. 1. Frecuencia del número de cromosomas, número modal en mitosis de $2 n=48$ (A) y en meiosis de $1 n=24$ (B) cromosomas en el robalo blanco $C$. undecimalis.

Fig. 1. Frequency of the number of chromosomes, modal number in mitosis with $2 n=48$ (A) and meiosis with $1 n=24$ (B) chromosomes from the common snook C. undecimalis. 
cromosómicos en meiosis quedó representado por la moda haploide de $1 \mathrm{n}=24$ cromosomas, misma que correspondió al 52.8\% (55 metafases) de campos observados (Fig. 1B). En el análisis de frecuencias de los campos meióticos fue evidente la presencia de células con $2 \mathrm{n}=48$ cromosomas que correspondió al $20.1 \%$ (21 metafases) del total de dispersiones cromosómicas analizadas. Por otro lado, se notaron algunas variaciones del número de cromosomas observados en estadio mitótico y meiótico, dicha variación osciló de $2 \mathrm{n}=40,44$ y de $1 n=20,27,41$ cromosomas respectivamente (Fig. 1A, B).

La longitud del complemento cromosómico promedio del cariotipo en mitosis de $C$. undecimallis, varió de $4.82 \pm 0.89 \mu \mathrm{m}$ en el primer par de cromosomas y hasta $1.62 \pm 0.27 \mu \mathrm{m}$ en ultimo par veinticuatro (Cuadro 1). Mientras, las medidas promedio del complemento cariotípico

CUADRO 1

Longitudes promedio en micrómetros $(\mu \mathrm{m})$ y relativas por par cromosómico del cariotipo en mitosis y meiosis del robalo blanco $C$. undecimalis

TABLE 1

Averaged and relative length of chromosome pairs in micrometers $(\mu \mathrm{m})$ from the karyotype in mitosis $(2 \mathrm{n}=48)$ and meiosis $(1 \mathrm{n}=24)$ of the common snook $C$. undecimalis

\begin{tabular}{|c|c|c|c|}
\hline Par cromosómico & $\begin{array}{l}\text { Longitud promedio de } \\
\quad \mathrm{q} \text { en } \mu \mathrm{m} \pm \mathrm{D} . \mathrm{E}\end{array}$ & $\begin{array}{l}\text { Longitud relativa promedio } \\
\qquad \text { de } \mathrm{q} \pm \mathrm{D} . \mathrm{E}\end{array}$ & Clasificación \\
\hline \multicolumn{4}{|c|}{ Cromosomas en mitosis $*$} \\
\hline 1 & $4.82 \pm 0.89^{a}$ & $6.44 \pm 0.10$ & \\
\hline 2 & $4.27 \pm 0.55^{\mathrm{a}}$ & $5.70 \pm 0.06$ & $\mathrm{~T}$ \\
\hline 3 & $4.08 \pm 0.46^{\mathrm{a}}$ & $5.46 \pm 0.05$ & $\mathrm{~T}$ \\
\hline 4 & $3.93 \pm 0.51^{\mathrm{a}}$ & $5.26 \pm 0.06$ & $\mathrm{~T}$ \\
\hline 5 & $3.82 \pm 0.48^{b}$ & $5.10 \pm 0.05$ & $\mathrm{~T}$ \\
\hline 6 & $3.67 \pm 0.52^{b}$ & $4.90 \pm 0.06$ & $\mathrm{~T}$ \\
\hline 7 & $3.55 \pm 0.46^{b}$ & $4.74 \pm 0.05$ & $\mathrm{~T}$ \\
\hline 8 & $3.50 \pm 0.46^{b}$ & $4.68 \pm 0.05$ & $\mathrm{~T}$ \\
\hline 9 & $3.47 \pm 0.46^{b}$ & $4.63 \pm 0.05$ & $\mathrm{~T}$ \\
\hline 10 & $3.30 \pm 0.50^{b}$ & $4.41 \pm 0.06$ & $\mathrm{~T}$ \\
\hline 11 & $3.13 \pm 0.41^{b}$ & $4.19 \pm 0.05$ & $\mathrm{~T}$ \\
\hline 12 & $3.10 \pm 0.39^{b}$ & $4.14 \pm 0.04$ & $\mathrm{~T}$ \\
\hline 13 & $3.01 \pm 0.48^{b}$ & $4.01 \pm 0.05$ & $\mathrm{~T}$ \\
\hline 14 & $2.95 \pm 0.47^{b}$ & $3.94 \pm 0.05$ & $\mathrm{~T}$ \\
\hline 15 & $2.88 \pm 0.40^{b}$ & $3.85 \pm 0.04$ & $\mathrm{~T}$ \\
\hline 16 & $2.83 \pm 0.39^{b}$ & $3.79 \pm 0.04$ & $\mathrm{~T}$ \\
\hline 17 & $2.70 \pm 0.50^{b}$ & $3.61 \pm 0.06$ & $\mathrm{~T}$ \\
\hline 18 & $2.65 \pm 0.51^{b}$ & $3.54 \pm 0.06$ & $\mathrm{~T}$ \\
\hline 19 & $2.53 \pm 0.49^{b}$ & $3.39 \pm 0.05$ & $\mathrm{~T}$ \\
\hline 20 & $2.47 \pm 0.38^{c}$ & $3.30 \pm 0.04$ & $\mathrm{~T}$ \\
\hline 21 & $2.35 \pm 0.43^{\mathrm{c}}$ & $3.14 \pm 0.05$ & $\mathrm{~T}$ \\
\hline 22 & $2.32 \pm 0.46^{\mathrm{c}}$ & $3.10 \pm 0.05$ & $\mathrm{~T}$ \\
\hline 23 & $1.88 \pm 0.27^{\mathrm{c}}$ & $2.52 \pm 0.03$ & $\mathrm{~T}$ \\
\hline 24 & $1.62 \pm 0.27 \mathrm{c}$ & $2.16 \pm 0.03$ & $\mathrm{~T}$ \\
\hline \multicolumn{4}{|c|}{ Cromosomas en meiosis $* *$} \\
\hline 1 & $2.60 \pm 0.46^{\mathrm{a}}$ & $7.54 \pm 0.04$ & $\mathrm{~T}$ \\
\hline 2 & $2.50 \pm 0.62^{\mathrm{a}}$ & $7.25 \pm 0.05$ & $\mathrm{~T}$ \\
\hline
\end{tabular}


CUADRO 1 (Continuación)

Longitudes promedio en micrómetros $(\mu \mathrm{m})$ y relativas por par cromosómico del cariotipo en mitosis y meiosis del robalo blanco $C$. undecimalis

TABLE 1 (Continued)

Averaged and relative length of chromosome pairs in micrometers $(\mu \mathrm{m})$ from the karyotype in mitosis $(2 \mathrm{n}=48)$ and meiosis $(1 \mathrm{n}=24)$ of the common snook $C$. undecimalis

\begin{tabular}{|c|c|c|c|}
\hline Par cromosómico & $\begin{array}{l}\text { Longitud promedio de } \\
\qquad \mathrm{q} \text { en } \mu \mathrm{m} \pm \mathrm{D} . \mathrm{E}\end{array}$ & $\begin{array}{l}\text { Longitud relativa promedio } \\
\qquad \text { de } q \pm D . E\end{array}$ & Clasificación \\
\hline 3 & $2.40 \pm 0.52^{\mathrm{a}}$ & $6.96 \pm 0.05$ & $\mathrm{~T}$ \\
\hline 4 & $2.30 \pm 0.69^{a}$ & $6.67 \pm 0.06$ & $\mathrm{~T}$ \\
\hline 5 & $1.90 \pm 0.46^{b}$ & $5.51 \pm 0.04$ & $\mathrm{~T}$ \\
\hline 6 & $1.90 \pm 0.46^{\mathrm{b}}$ & $5.51 \pm 0.04$ & $\mathrm{~T}$ \\
\hline 7 & $1.70 \pm 0.46^{\mathrm{b}}$ & $4.93 \pm 0.04$ & $\mathrm{~T}$ \\
\hline 8 & $1.70 \pm 0.46^{\mathrm{b}}$ & $4.93 \pm 0.04$ & $\mathrm{~T}$ \\
\hline 9 & $1.50 \pm 0.30^{b}$ & $4.35 \pm 0.03$ & $\mathrm{~T}$ \\
\hline 10 & $1.50 \pm 0.30^{b}$ & $4.35 \pm 0.03$ & $\mathrm{~T}$ \\
\hline 11 & $1.40 \pm 0.46^{b}$ & $4.06 \pm 0.04$ & $\mathrm{~T}$ \\
\hline 12 & $1.40 \pm 0.46^{\mathrm{b}}$ & $4.06 \pm 0.04$ & $\mathrm{~T}$ \\
\hline 13 & $1.30 \pm 0.35^{b}$ & $3.77 \pm 0.03$ & $\mathrm{~T}$ \\
\hline 14 & $1.20 \pm 0.30^{\mathrm{b}}$ & $3.48 \pm 0.03$ & $\mathrm{~T}$ \\
\hline 15 & $1.20 \pm 0.30^{b}$ & $3.48 \pm 0.03$ & $\mathrm{~T}$ \\
\hline 16 & $1.20 \pm 0.30^{b}$ & $3.48 \pm 0.03$ & $\mathrm{~T}$ \\
\hline 17 & $1.10 \pm 0.17^{\mathrm{b}}$ & $3.19 \pm 0.02$ & $\mathrm{~T}$ \\
\hline 18 & $1.10 \pm 0.17^{b}$ & $3.19 \pm 0.02$ & $\mathrm{~T}$ \\
\hline 19 & $1.00 \pm 0.35^{\mathrm{c}}$ & $2.90 \pm 0.03$ & $\mathrm{~T}$ \\
\hline 20 & $1.00 \pm 0.35^{\mathrm{c}}$ & $2.90 \pm 0.03$ & $\mathrm{~T}$ \\
\hline 21 & $0.90 \pm 0.30^{\mathrm{c}}$ & $2.61 \pm 0.03$ & $\mathrm{~T}$ \\
\hline 22 & $0.90 \pm 0.30^{c}$ & $2.61 \pm 0.03$ & $\mathrm{~T}$ \\
\hline 23 & $0.80 \pm 0.17^{\mathrm{c}}$ & $2.32 \pm 0.02$ & $\mathrm{~T}$ \\
\hline 24 & $0.80 \pm 0.17^{c}$ & $2.32 \pm 0.02$ & $\mathrm{~T}$ \\
\hline
\end{tabular}

Discrepancias entre los superíndices y símbolos indican diferencia significativa en el estadístico de Tukey $\mathrm{p}<0.05$, q=brazo largo; D.E=desviación estándar, T=telocéntrico.

Discrepancy between superscript and symbols indicates significant difference under Tukey test $\mathrm{p}<0.05$, $\mathrm{q}=$ long arm; D.E $=$ standard deviation, $\mathrm{T}=$ telocentric.

haploide bivalente en meiosis, mostró en el primer par cromosómico $2.60 \pm 0.46 \mu \mathrm{m}$ y en el último par veinticuatro $0.80 \pm 0.17 \mu \mathrm{m}$ (Cuadro 1). El analisis de varianza, mostró que existen diferencias significativas entre las longitudes promedio de los pares cromosómicos, mismos que fueron clasificados en tres grupos basado en las diferencias estadísticas $(\mathrm{p}<0.05)$ identificadas, como sigue: cromosomas mayores (par 1-4), medios (par 5-19) y menores (par 20-24) del cariotipo promedio en mitosis (Cuadro 1). El complemento cariotípico promedio en meiosis, se clasificó en grupos similares al del cariotipo en mitosis y solo con variaciones en los número de pares correspondientes, como se muestra a continuación: cromosomas mayores (par 1-4), medios (par 5-20) y menores (par 21-24) (Cuadro 1). Adicionalmente, el analisis de varianza, permitió el establecimiento de diferencias significativas entre las medidas promedio de los pares cromosómicos del cariotipo global entre la mitosis y meiosis (Cuadro 1). 
El cariotipo característico del robalo blanco $C$. undecimalis tuvo como N.F a 48 brazos cromosómicos y fue clasificado como primitivo por estar integrado en estadio mitótico $(2 \mathrm{n}=48)$ y meiótico $(1 \mathrm{n}=24)$ por cromosomas monorrameos de tipo telocéntrico (T) (Fig. 2). El ideograma del complemento cromosómico promedio por par cromosómico de los cariotipos en mitosis y meiosis reflejan esquemáticamente las diferencias en longitud $(\mu \mathrm{m})$ entre ambos estadios celulares. Comparativamente, dichas diferencias representan aproximadamente el doble de las longitudes en micrometros observadas en el cariotipo en mitosis cuando éste se contrasta con el del estadio en meiosis (Fig. 3). Por otro lado, no se lograron observar diferencias en tamaño y forma entre los cromosomas que integran las dispersiones cromosómicas de hembras y machos de la especie analizada.

\section{DISCUSIÓN}

En los estudios de citogenética, que se han realizado en varios grupos taxonómicos que integran a los peces se ha observado con

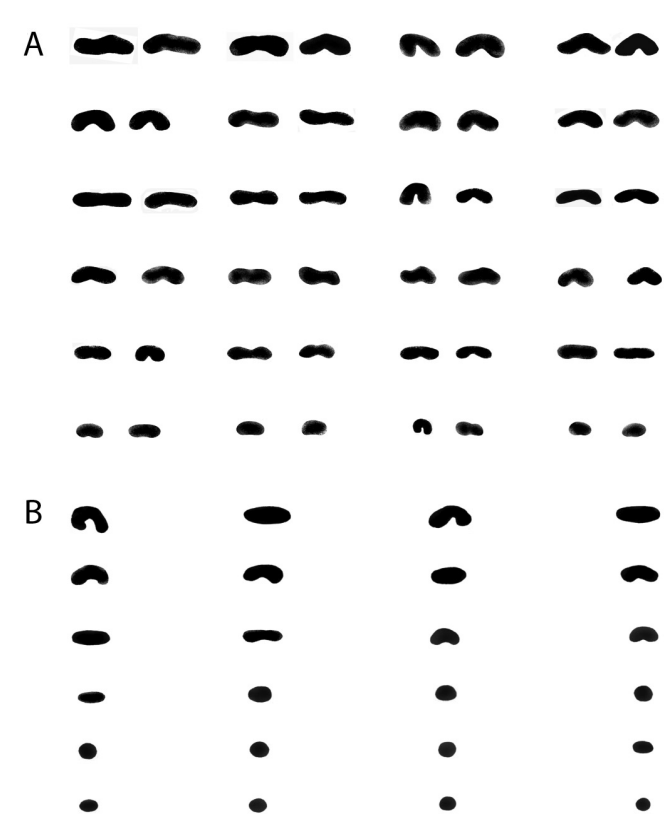

Fig. 2. Cariotipo típico diploide (A) con $2 n=48$ cromosomas telocéntricos (T) y cariotipo haploide (B) con $1 \mathrm{n}=24$ cromosomas (T) de C. undecimalis.

Fig. 2. Typical diploid karyotype (A) in mitosis with $2 n=48$ telocentric $(\mathrm{T})$ chromosomes and haploid karyotype (B) with $1 \mathrm{n}=24$ chromosomes $(\mathrm{T})$ of $C$. undecimalis.

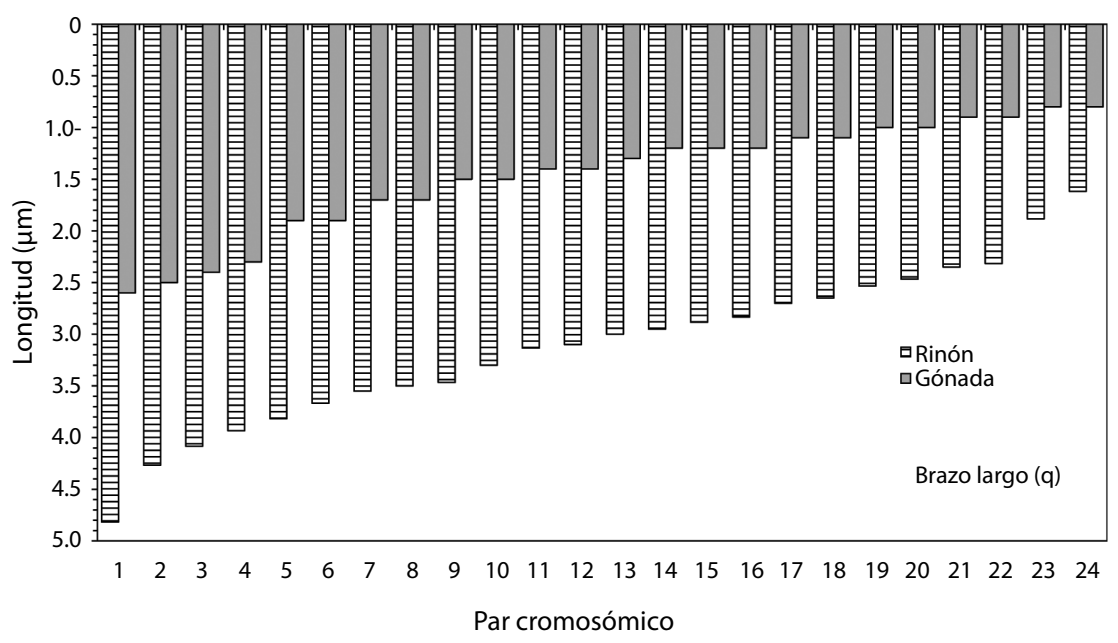

Fig. 3. Ideograma representativo del complemento cromosómico promedio por pares, del cariotipo en mitosis (barras obscuras) y en meiosis (barras claras) de $C$. undecimalis.

Fig. 3. Representative ideogram from the averaged chromosome complement by pairs of the karyotype in mitosis (dark bars) and meiosis (clear bars) of C. undecimalis. 
frecuencia un efecto diferencial del alcaloide colchicina sobre los órganos y tejidos blancos que son ricos principalmente en células epiteliales con alto ritmo de divisiones celulares (Denton \& Howell 1969, Denton 1973, Kligerman \& Bloom 1977, Gold 1979, Ramírez 1980, Thorgaard \& Disney 1990). Dicho efecto diferencial se ha observado por presencia de dispersiones cromosómicas o metafases en sólo algunos de los tejidos del pez (p.e. tejido branquial, riñón y gónadas) (Denton \& Howell 1969, Denton 1973, Kligerman \& Bloom 1977, Gold 1979, Ramírez 1980, Thorgaard \& Disney 1990). En el caso de la especie de que trata este estudio, sólo fue posible observar dispersiones cromosómicas de calidad y con número suficiente en el tejido riñón y gónadas, probablemente por que las células epiteliales de las branquias presentan efecto refractario hacia el alcaloide colchicina (razón por la que no fue posible observar metafases a partir del tejido branquial) como ha sido observado en otras especies de peces para otros tejidos (Denton \& Howell 1969, Denton 1973, Kligerman \& Bloom 1977, Gold 1979, Ramírez 1980, Thorgaard \& Disney 1990).

El número cromosómico de $2 \mathrm{n}=48$ y $1 \mathrm{n}=24$, identificado para el robalo blanco $C$. undecimalis, es similar al estudio de BarretoNeto (2001), para C. mexicanus y $C$. undecimalis. El número cromosómico reportado para los Centropomidos por Barreto-Neto (2001) $\mathrm{y}$ el presente estudio de $2 \mathrm{n}=48 \quad$ (y $1 \mathrm{n}=24$ ) cromosomas coinciden con el valor de $2 n=48$ cromosomas, propuesto como número cromosómico ancestral para la mayoría de peces perciformes (Denton 1973, Ohno 1974, Galetti Jr. et al. 2000). Por otro lado, varios estudios recientes en peces marinos han demostrado que la mayoría de especies analizadas desde el punto de vista citogenético muestran $2=48$ cromosomas monorrámeos de tipo telocéntrico (T) (Galetti Jr. et al. 2000, Ruiz-Carus 2002, Accioly \& Molina 2008); por lo que se ha considerado dicho carácter citogenético, como una condición biológica que es compartida por los peces marinos.
La presencia de cromosomas sexuales en peces, es una condición biológica que se ha observado muy pocas veces en alrededor de 7000 especies vivientes con hábitat marino (Galetti Jr. et al. 2000, Ueno \& Takai 2008). En el caso de $C$. undecimalis, no fue posible identificar heteromorfismo cromosómico entre las dispersiones cromosómicas de hembras y de machos, por ello se descarta la presencia de cromosomas sexuales en la especie y probablemente dicha condición es compartida por el resto de especies que comprenden a la familia Centropomidae. La constitución cromosómica observada, también sugiere que la determinación sexual en el robalo blanco no se establece al momento de la fertilización (singamia) por heterocromosomas. Dicha condición biológica permite que el cigoto tenga el potencial de desarrollarse en hembra o macho (o inversamente) en cualquiera de las etapas del ciclo de vida, siguiendo un patrón similar al de una dosis hormonal compensatoria, basada en una represión transcriptacional parcial de genes que regulan el sexo (Dawes et al. 1999). El aspecto práctico de la conjetura anterior ha sido también observada en dos especies de peces serranidos (Epinephelus guttatus y Thalassoma bifasciatum) (Ruiz-Carus 2002). En el robalo blanco, los especímenes silvestres durante las primeras etapas de vida primero maduran como machos y posteriormente se desarrollan hacia hembras (Peters et al. 1998). El potencial dado por la carencia de cromosomas sexuales, se ha comprobado en experimentos de reversión sexual hacia hembras de juveniles macho de $C$. undecimalis empleando el esteroide 17- $\beta$ estradiol (Vidal-López 2009). Sin embargo, estudios adicionales de genética básica siguen siendo importantes para el buen manejo del recurso y su aplicación en sistemas de cultivo.

\section{AGRADECIMIENTOS}

El presente documento tuvo financiamiento del proyecto de repatriación 76305 (CONACYT) otorgado al primer autor (LAR). Los autores agradecen a Ulises Hernández 
Vidal (DACBiol-UJAT) y Juan M. Vidal López (DACBiol-UJAT) por el apoyo brindado.

\section{RESUMEN}

El robalo blanco Centropomus undecimalis, vive en hábitats marinos, salobres y dulceacuícolas en el océano Atlántico occidental, incluyendo el golfo de México. La especie, es económicamente importante en varias localidades, no obstante los estudios sobre su biología y genética son hasta el momento pocos. El presente estudio tiene como propósito, la caracterización citogenética de especímenes recolectados en el municipio de Paraíso, Tabasco, México. Cinco hembras y ocho machos fueron procesados por técnicas citológicas convencionales para la obtención de preparaciones cromosómicas de buena calidad para elaborar el cariotipo. Los resultados del análisis del tejido del riñón, mostraron que $85.1 \%$ de 288 mitosis tienen $2 \mathrm{n}=48$ cromosomas y $52.8 \%$ de 104 meiosis exhiben el número haploide de $1 \mathrm{n}=24$. El cariotipo diploide mostro 48 cromosomas monorrámeos de tipo telocéntrico $(\mathrm{T})$. No se observó heteromorfismo cromosómico entre hembras y machos. El cariotipo diploide fue similar a los observados en la mayoría de peces marinos.

Palabras clave: cariotipo, cromosoma, robalo blanco, Centropomus undecimalis.

\section{REFERENCIAS}

Accioly, I.V. \& W.F. Molina. 2008. Cytogenetic studies in Brazilian marine Sciaenidae and Sparidae fishes (Perciformes). Genet. Mol. Res. 2: 358-70.

Álvarez-Lanjonchére, L. \& T.M. Suzuki. 2008. A review of methods for Centropomus spp. (snooks) aquaculture and recommendations for the establishment of their culture in Latin America. Aquaculture Res. 39: 684-700.

Arias-Rodriguez, L., J.P. González-Hermoso, H. FletesRegalado, L.E. Rodríguez-Ibarra \& G. Del Valle Pignataro. 2007. Cariotipos de los caracoles de tinte Plicopurpura pansa (Gould, 1853) y Plicopurpura columellaris (Lamarck, 1816) (Gastropoda: Muricidae). Rev. Biol. Trop. 55: 853-866.

Arias-Rodriguez, L., L. Ibarra-Castro \& S. Páramo-Delgadillo. 2008. Los cromosomas mitóticos y meióticos del pez tropical Petenia splendida (Pisces: Cichlidae). Rev. Biol. Trop. 56: 895-907.

Arias-Rodriguez, L., S. Páramo-Delgadillo, W.M. Contreras-Sánchez \& C.A. Álvarez-González. 2009. Cariotipo del pejelagarto tropical Atractosteus tropicus (Lepisosteiformes: Lepisosteidae) y variación cromosómica en sus larvas y adultos. Rev. Biol. Trop. 57: 529-539.

Barreto-Netto, M.R.C. 2001. Caracterizacao citogenetica e molecular de duas espécies do gênero Centropomus (Teleostei, Perciformes). Tesis de Maestría, Universidade Estadual Paulista Júlio de Mesquita Filho, Brasil.

Chávez, H. 1963. Contribución al conocimiento de la biología de los robalos, chucumite y Constantino Centropomus sp del estado de Veracruz. Ciencia 22: 141-161.

Dawes, H.E., D.S. Berlin, D.M. Lapidus, C. Nusbaum, T.L. Davis \& B.J. Meyer. 1999. Dosage compensation proteins targeted to $\mathrm{X}$ chromosomes by a determinant of hermaphrodite fate. Science 284: 1800-1804.

Denton, T.E. 1973. Fish chromosome methodology. Charles, Illinois, EEUU.

Denton, T.E. \& W.M. Howell. 1969. A technique for obtaining chromosomes from the scale epithelium of teleost fishes. Copeia 2: 392-393.

Galetti Jr. Jr., P.M., C.T. Aguilar \& W.F. Molina. 2000. An overview on marine fish cytogenetics. Hydrobiologia 420: 55-62.

Gold, J.R. 1979. Cytogenetics, p. 353-405. In W.S. Hoar, D.J. Randall \& J.R. Brett. Fish physiology. Bioenergetics and growth. Academic, Florida, EEUU.

Kligerman, A.D. \& S.E. Bloom. 1977. Rapid chromosome preparations from solid tissues of fishes. J. Fish. Res. Board Can. 34: 266-269.

Levan, A., K. Fredga \& A.A. Sandberg. 1964. Nomenclature for centromeric position on chromosomes. Hereditas 52: 201-220.

Marshall, A.R. 1958. A survey of snook fisheries of Florida, with studies of the biology of the principal species, Centropomus undecimalis (Bloch). Florida. State Bd. Conser. Tech. Ser. 22: 5-37.

Miller, R.R., W.L. Mincley \& S.M. Norris. 2005. Freshwater fishes of México. University of Chicago, Chicago, EEUU.

Nelson, J.S. 2006. Fishes of the world. Wiley, Nueva York, EEUU.

Ohno, S. 1974. Protochordata, Clyclostomata and pisces, p. 1-92. In B. John. Animal cytogenetics. Borntraeger, Berlin-Stuttgart, Alemania. 
Peregrino-Uriarte, A.B., R. Pacheco-Aguilar, A. RomeroVarela \& G. Yepiz-Plascencia. 2007. Diferencias en los genes 16SrRNA y citocromo C oxidasa subunidad I de las lisas Mugil cephalus y Mugil curema y los robalos Centropomus viridis y Centropomus robalito. Cien. Mar. 33: 95-104.

Perera-García, M.A., M. Mendoza-Carranza \& S. Páramo-Delgadillo. 2008. Dinámica reproductiva y poblacional del robalo, Centropomus undecimalis (Perciformes: Centropomide) en barra San Pedro, Centla, México. Universidad y Ciencia 24: 49-59.

Peters, K.M., R.E. Matheson Jr. \& R.G. Taylor. 1998. Reproduction and early life history of common snook, Centropomus undecimalis (Bloch), in Florida. Bull. Mar. Sci. 63: 509-529.

Ramirez, S.A. 1980. A modified technique for fish karyotype analysis using scale epithelium. Copeia 3 : 543-545.

Rivas, L.R. 1986. Systematic review of the Perciform fishes of the genus Centropomus. Copeia 3: 579-611.

Ruiz-Carus, R. 2002. Chromosome analysis of the sexual phases of the protogynous hermaphrodites Epinephelus guttatus and Thalassoma bifasciatum (Serranidae and Labridae; Teleostei). Caribb. J. Sci. 38: 44-51.
Thorgaard, G.H. \& J.E. Disney. 1990. Chromosome preparation and analysis, p. 171-190. In C.B. Schreck \& P.B. Moyle. Methods for fish biology. American Fisheries Society, Bethesda, Maryland.

Tringali, M.D., T.M. Bert \& S. Seyoum. 1999. Genetic identification of Centropomidae fishes. Trans. Am. Fish. Soc. 128: 446-458.

Tucker Jr., J.W. 1987. Snook and tarpon snook culture and preliminary evaluation for commercial farming. Prog. Fish. Cult. 49: 49-57.

Ueno, K. \& A. Takai. 2008. Multiple sex chromosome system of $\mathrm{X}_{1} \mathrm{X}_{1} \mathrm{X}_{2} \mathrm{X}_{2} / \mathrm{X}_{1} \mathrm{X}_{2} \mathrm{Y}$ type in lutjanid fish, Lutjanus quinquelineatus (Perciformes). Genetica 132: $35-41$.

Vidal-López, J.M. 2009. Efecto de la temperatura y el esteroide 17- $\beta$ estradiol sobre la proporción de sexos en juveniles de robalo blanco Centropomus undecimalis. Tesis de Maestría, Universidad Juárez Autónoma de Tabasco, Tabasco, México.

Volpe, A.V. 1959. Aspects of the biology of the common snook Centropomus undecimalis (Bloch), of southwest Florida. Florida. State Bd. Conser. Tech. Ser. 31: 7-36.

Zar, J.H. 1984. Biostatistical analysis. Prentice, Nueva Jersey, EEUU. 